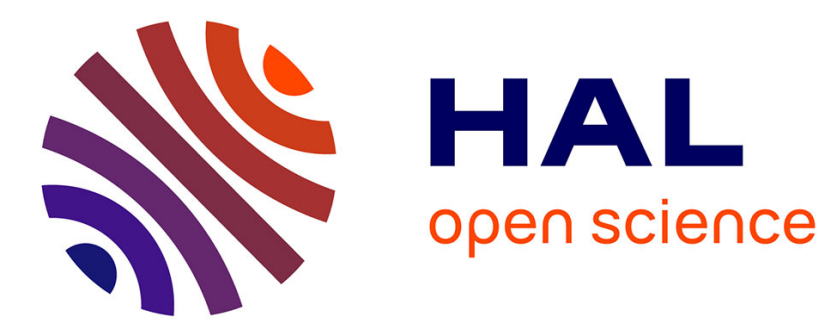

\title{
MAC-BC duality with linear-feedback schemes
}

Selma Belhadj Amor, Yossef Steinberg, Michèle Wigger

\section{To cite this version:}

Selma Belhadj Amor, Yossef Steinberg, Michèle Wigger. MAC-BC duality with linear-feedback schemes. 2014 IEEE International Symposium on Information Theory (ISIT), Jun 2014, Honolulu, United States. 10.1109/ISIT.2014.6875131 . hal-01221220

\section{HAL Id: hal-01221220 \\ https://hal.science/hal-01221220}

Submitted on 27 Oct 2015

HAL is a multi-disciplinary open access archive for the deposit and dissemination of scientific research documents, whether they are published or not. The documents may come from teaching and research institutions in France or abroad, or from public or private research centers.
L'archive ouverte pluridisciplinaire HAL, est destinée au dépôt et à la diffusion de documents scientifiques de niveau recherche, publiés ou non, émanant des établissements d'enseignement et de recherche français ou étrangers, des laboratoires publics ou privés. 


\section{MAC-BC Duality with Linear-Feedback Schemes}

\author{
Selma Belhadj Amor \\ Telecom ParisTech \\ Paris, France \\ belhadjamor@telecom-paristech.fr
}

\author{
Yossef Steinberg \\ Technion-Israel Inst. of Technology \\ Haifa, Israel \\ ysteinbe@ee.technion.ac.il
}

\author{
Michèle Wigger \\ Telecom ParisTech \\ Paris, France \\ wigger@telecom-paristech.fr
}

\begin{abstract}
We show that for the multi-antenna Gaussian MAC and $\mathrm{BC}$ with perfect feedback, the largest achievable regions with linear-feedback schemes (called linear-feedback capacity regions) coincide when the same total input-power constraint is imposed on both channels and when the MAC channel matrices are the transposes of the $\mathrm{BC}$ channel matrices.

In the two-user case, when either transmitters or receiver are single-antenna, the capacity region for the Gaussian MAC is known and the capacity-achieving scheme is a linear-feedback scheme. With our results we can thus determine the linearfeedback capacity region of the two-user Gaussian $\mathrm{BC}$ when either transmitter or receivers are single-antenna. For these cases we can also identify the linear-feedback schemes that achieve the linear-feedback capacity.

Our results also extend to a partial-feedback setup where only a subset of the feedback links are present.
\end{abstract}

\section{INTRODUCTION}

We study the multi-input multi-output (MIMO) Gaussian multi-access channel (MAC) and broadcast channel (BC) with perfect output feedback, where the transmitter(s) observe(s) the channel outputs in a noise-free but causal way. It is well known that feedback can increase capacity for these channels. The exact capacity regions however are generally unknown.

In this paper we restrict attention to a specific class of coding schemes, called linear-feedback schemes [1], where the tansmitter(s) can use the feedback signals only in a linear way. More specifically, in every channel use, the produced inputs are restricted to linear combinations of the past feedback signals and some information-carrying code symbols which only depend on the messages available at the corresponding transmitter. The best coding schemes known to date both for the MIMO Gaussian MAC and BC with perfect feedback [2], [3], [4], [5], [6], [7], [8], [9] are linear-feedback schemes. For the two-user single-input single-output (SISO), the twouser single-input multi-output (SIMO), and the two-user multiinput single-output (MISO) Gaussian MAC [2], [3] linearfeedback schemes even achieve the entire capacity region. (Notice that also the rate-splitting extensions of the schemes in [2] and [3] are linear-feedback schemes.)

Without feedback, the following duality relation is well known [10], [11], [12]: under the same total input-power constraint the capacity regions of the MIMO Gaussian MAC and $\mathrm{BC}$ coincide when the channel matrices of the MAC and $\mathrm{BC}$ are transposes of each other. Such a pair of MAC and $\mathrm{BC}$ is called dual. We have recently shown a similar duality relation for the SISO Gaussian MAC and BC with perfect feedback when one restricts to linear-feedback schemes [13].
Our main result in this paper is the following new duality result: with perfect feedback and when restricting to linearfeedback schemes, the set of all achievable rates, called linearfeedback capacity regions, coincide for the MIMO Gaussian MAC and BC when the two channels are dual and when the same total input-power constraint is imposed on both channels. This result is particularly interesting for the two-user SISO, SIMO, and MISO cases where computable single-letter characterizations of the linear-feedback capacity regions of the Gaussian MAC are known. With our duality result, we thus immediately obtain single-letter characterizations of the linearfeedback capacity regions for the two-user SISO, SIMO, and MISO Gaussian BC.

We further introduce a class of (multi-letter) linear-feedback schemes for the MIMO Gaussian MAC and BC that achieve the linear-feedback capacity. Within this class we then identify the pairs of schemes that achieve the same rate-regions over dual MACs and BCs. Since we know the best linear-feedback schemes for the two-user SISO, SIMO, and MISO MAC [2], [3], we can identify the best linear-feedback schemes for the two-user SISO, SIMO, and MISO BC.

For clarity of exposition, in what follows we consider only two users. All our results extend to arbitrary $K \geq 2$ number of users. Moreover, our results also extend to partial-feedback setups where only a subset of the feedback links are present.

\section{Mimo Gaussian BC with Feedback}

We consider the two-user memoryless MIMO Gaussian BC with perfect feedback. The transmitter is equipped with $\kappa$ transmit-antennas and each Receiver $i$, for $i \in\{1,2\}$, with $\nu_{i}$ receive-antennas. At each discrete time $t$, if $\mathbf{x}_{t}$ denotes the real vector-valued input symbol sent by the transmitter, Receiver $i$ observes the real vector-valued channel output

$$
\mathbf{Y}_{i, t}=\mathrm{H}_{i} \mathbf{x}_{t}+\mathbf{Z}_{i, t}
$$

where $\mathrm{H}_{i}$, for $i \in\{1,2\}$, is a deterministic real $\nu_{i}$-by- $\kappa$ channel matrix known to transmitter and receivers and $\left\{\mathbf{Z}_{1, t}\right\}_{t=1}^{n}$ and $\left\{\mathbf{Z}_{2, t}\right\}_{t=1}^{n}$ are independent sequences of independent and identically distributed (i.i.d.) centered $\kappa$-dimensional Gaussian random vectors of identity covariance matrix.

The goal of the communication is that the transmitter conveys Message $M_{1}$ to Receiver 1 and Message $M_{2}$ to Receiver 2 . The messages are independent of the noise sequences $\left\{\mathbf{Z}_{1, t}\right\}_{t=1}^{n}$ and $\left\{\mathbf{Z}_{2, t}\right\}_{t=1}^{n}$ and uniformly distributed over the sets $\mathcal{M}_{1} \triangleq\left\{1, \ldots,\left\lfloor 2^{n R_{1}}\right\rfloor\right\}$ and $\mathcal{M}_{2} \triangleq\left\{1, \ldots,\left\lfloor 2^{n R_{2}}\right\rfloor\right\}$, 
where $R_{1}$ and $R_{2}$ denote the rates of transmission and $n$ the blocklength. The transmitter observes causal, noise-free output feedback from both receivers. Thus, the time- $t \in\{1, \ldots, n\}$ channel input $\mathbf{X}_{t}$ can depend on the messages $M_{1}$ and $M_{2}$ and the channel outputs $\mathbf{Y}_{1,1}, \ldots, \mathbf{Y}_{1, t-1}$ and $\mathbf{Y}_{2,1}, \ldots, \mathbf{Y}_{2, t-1}$ :

$\mathbf{X}_{t}=\varphi_{t}^{(n)}\left(M_{1}, M_{2}, \mathbf{Y}_{1,1}, \ldots, \mathbf{Y}_{1, t-1}, \mathbf{Y}_{2,1}, \ldots, \mathbf{Y}_{2, t-1}\right)$,

for some encoding function of the form $\varphi_{t}^{(n)}: \mathcal{M}_{1} \times \mathcal{M}_{2} \times$ $\mathbb{R}^{\nu_{1}(t-1)} \times \mathbb{R}^{\nu_{2}(t-1)} \rightarrow \mathbb{R}^{\kappa}$.

We impose an expected average block-power constraint

$$
\frac{1}{n} \sum_{t=1}^{n} \mathbf{E}\left[\left\|\mathbf{X}_{t}\right\|^{2}\right] \leq P .
$$

Each Receiver $i$ decodes its corresponding message $M_{i}$ by means of a decoding function $\phi_{i}^{(n)}$ of the form $\phi_{i}^{(n)}: \mathbb{R}^{\nu_{i} n} \rightarrow$ $\mathcal{M}_{i}$, for $i \in\{1,2\}$. That means, based on the output sequence $\mathbf{Y}_{i}^{n}$, Receiver $i$ produces the guess $\hat{M}_{i}^{(n)}=\phi_{i}^{(n)}\left(\mathbf{Y}_{i}^{n}\right)$. The average probability of error is

$$
P_{\mathrm{e}, \mathrm{BC}}^{(n)} \triangleq \operatorname{Pr}\left[\left(\hat{M}_{1}, \hat{M}_{2}\right) \neq\left(M_{1}, M_{2}\right)\right] .
$$

A rate-pair $\left(R_{1}, R_{2}\right)$ is achievable over the MIMO Gaussian BC with feedback under a power constraint $P$, if there exists a sequence of encoding and decoding functions $\left\{\left\{\varphi_{t}^{(n)}\right\}_{t=1}^{n}, \phi_{1}^{(n)}, \phi_{2}^{(n)}\right\}_{n=1}^{\infty}$, as described above, satisfying the power constraint (3) and such that the average probability of error $P_{\mathrm{e}, \mathrm{BC}}^{(n)}$ tends to zero as $n$ tends to infinity. The closure of the union of all achievable regions is called capacity region.

In the present paper, we focus on linear-feedback coding schemes where the transmitter's channel input is a linear combination of the previous feedback output signals and an information-carrying vector that depends only on the messages $\left(M_{1}, M_{2}\right)$ (but not on the feedback). Specifically, we assume that the channel input vectors have the form

$$
\mathbf{X}_{t}=\mathbf{V}_{t}+\sum_{i=1}^{2} \sum_{\tau=1}^{t-1} \mathrm{~A}_{i, \tau, t} \mathbf{Y}_{i, \tau}, \quad t \in\{1, \ldots, n\},
$$

where $\left\{\mathrm{A}_{i, \tau, t}\right\}$ are arbitrary $\kappa$-by- $\nu_{i}$ matrices and $\mathbf{V}_{t}=$ $\xi_{t}^{(n)}\left(M_{1}, M_{2}\right)$ for some function $\xi_{t}^{(n)}: \mathcal{M}_{1} \times \mathcal{M}_{2} \rightarrow \mathbb{R}^{\kappa}$.

The set of all rate-pairs achieved by linear-feedback schemes is called linear-feedback capacity region and is denoted $\mathcal{C}_{\mathrm{BC}}^{\text {linfb }}\left(\mathrm{H}_{1}, \mathrm{H}_{2} ; P\right)$. The largest sum-rate achieved by a linear-feedback scheme is called linear-feedback sum-capacity and is denoted $C_{\mathrm{BC}, \Sigma}^{\text {linfb }}\left(\mathrm{H}_{1}, \mathrm{H}_{2} ; P\right)$.

\section{MiMO Gaussian MAC with FeEdback}

We consider the two-user memoryless MIMO Gaussian MAC with perfect feedback. Each Transmitter $i$, for $i \in\{1,2\}$, is equipped with $\nu_{i}$ transmit-antennas and the receiver with $\kappa$ receive-antennas. At each discrete time $t$, if $\mathbf{x}_{1, t}$ and $\mathbf{x}_{2, t}$ denote the vector signals sent by Transmitters 1 and 2, the receiver observes the real vector-valued channel output

$$
\mathbf{Y}_{t}=\mathbf{H}_{1}^{\top} \mathbf{x}_{1, t}+\mathbf{H}_{2}^{\top} \mathbf{x}_{2, t}+\mathbf{Z}_{t},
$$

where $\mathrm{H}_{i}$, for $i \in\{1,2\}$, is a deterministic real $\nu_{i}$-by- $\kappa$ channel matrix known to transmitters and receiver and $\left\{\mathbf{Z}_{t}\right\}$ is a sequence of i.i.d. $\kappa$-dimensional centered Gaussian random vectors of identity covariance matrix.

The goal of communication is that Transmitters 1 and 2 convey independent messages $M_{1}$ and $M_{2}$ (which are also independent of $\left.\left\{\mathbf{Z}_{t}\right\}\right)$ to the common receiver. Since the transmitters have perfect feedback, Transmitter $i$ 's, $i \in\{1,2\}$, channel input at time $t, \mathbf{X}_{i, t}$, can depend on its message $M_{i}$ and the previous output vectors $\mathbf{Y}_{1}, \ldots, \mathbf{Y}_{t-1}$ :

$$
\mathbf{X}_{i, t}=\varphi_{i, t}^{(n)}\left(M_{i}, \mathbf{Y}_{1}, \ldots, \mathbf{Y}_{t-1}\right), \quad t \in\{1, \ldots, n\},
$$

for some encoding functions $\varphi_{i, t}^{(n)}: \mathcal{M}_{i} \times \mathbb{R}^{\kappa(t-1)} \rightarrow \mathbb{R}$.

The channel input sequences $\left\{\mathbf{X}_{1, t}\right\}_{t=1}^{n}$ and $\left\{\mathbf{X}_{2, t}\right\}_{t=1}^{n}$ have to satisfy a total expected average block-power constraint $P$ :

$$
\frac{1}{n} \sum_{t=1}^{n}\left(\mathbf{E}\left[\left\|\mathbf{X}_{1, t}\right\|^{2}\right]+\mathbf{E}\left[\left\|\mathbf{X}_{2, t}\right\|^{2}\right]\right) \leq P .
$$

The receiver decodes the messages $\left(M_{1}, M_{2}\right)$ by means of a decoding function $\phi^{(n)}$ of the form $\phi^{(n)}: \mathbb{R}^{\kappa n} \rightarrow \mathcal{M}_{1} \times \mathcal{M}_{2}$. This means, based on the output sequence $\mathbf{Y}_{1}, \ldots, \mathbf{Y}_{n}$, the receiver produces its guess $\left(\hat{M}_{1}, \hat{M}_{2}\right)=\phi^{(n)}\left(\mathbf{Y}_{1}, \ldots, \mathbf{Y}_{n}\right)$. The average probability of error is defined as

$$
P_{\mathrm{e}, \mathrm{MAC}}^{(n)} \triangleq \operatorname{Pr}\left[\left(\hat{M}_{1}, \hat{M}_{2}\right) \neq\left(M_{1}, M_{2}\right)\right]
$$

A rate-pair $\left(R_{1}, R_{2}\right)$ is achievable over the Gaussian MIMO MAC with feedback under a sum-power constraint $P$, if there exists a sequence of encoding and decoding functions $\left\{\left\{\varphi_{1, t}^{(n)}\right\}_{t=1}^{n},\left\{\varphi_{2, t}^{(n)}\right\}_{t=1}^{n}, \phi^{(n)}\right\}_{n=1}^{\infty}$, as described above, such that the average probability of a decoding error $P_{\mathrm{e}, \mathrm{MAC}}^{(n)}$ tends to zero as $n$ tends to infinity. The closure of the union of all achievable regions is called capacity region.

Also here, we focus on the class of linear-feedback coding schemes where the channel inputs at Transmitter $i$, for $i \in\{1,2\}$, are given by linear combinations of the previous feedback signals and an information-carrying vector that only depends on the message $M_{i}$ (but not on the feedback). Specifically, we assume that the channel input vectors satisfy

$$
\mathbf{X}_{i, t}=\mathbf{U}_{i, t}+\sum_{\tau=1}^{t-1} \mathrm{C}_{i, \tau, t} \mathbf{Y}_{\tau}, \quad t \in\{1, \ldots, n\},
$$

where $\left\{\mathrm{C}_{i, \tau, t}\right\}$ are arbitrary $\nu_{i}$-by- $\kappa$ matrices and $\mathbf{U}_{i, t}=$ $\xi_{i, t}^{(n)}\left(M_{i}\right)$ for an arbitrary encoding function $\xi_{i, t}^{(n)}: \mathcal{M}_{1} \rightarrow \mathbb{R}^{\nu_{i}}$.

Linear-feedback capacity and linear-feedback sum-capacity are defined analougously to the $\mathrm{BC}$. We denote them by $\mathcal{C}_{\mathrm{MAC}}^{\text {linfb }}\left(\mathrm{H}_{1}^{\top}, \mathrm{H}_{2}^{\top} ; P\right)$ and $C_{\mathrm{MAC}, \Sigma}^{\text {linfb }}\left(\mathrm{H}_{1}^{\top}, \mathrm{H}_{2}^{\top} ; P\right)$.

\section{DUAL SCHEMES FOR MAC AND BC AND LINEAR-FEEDBACK CAPACITIES}

The idea of our schemes is to divide the blocklength $n$ into $n^{\prime}$ subblocks of equal length $\eta$. An inner code uses the feedback to transform each subblock of $\eta$ channel uses of the original MIMO BC or MAC into a single channel use of a new MIMO BC or MAC, with more transmit and receive antennas. An outer code is then applied to communicate over the new MIMO BC or MAC without using the feedback. 


\section{A. A class of linear-feedback schemes for the BC}

Fix the blocklength $n$. Our schemes are parametrized by

- a positive integer $\eta$;

- $\kappa$-by- $\nu_{i}$ matrices $\left\{\mathrm{A}_{i, \tau, \ell}\right\}$, for $\ell=2, \ldots, \eta, \tau=$ $1, \ldots, \ell-1$, and $i \in\{1,2\}$;

- an encoding mapping $f^{\left(n^{\prime}\right)}: \mathcal{M}_{1} \times \mathcal{M}_{2} \rightarrow\left(\mathcal{R}^{\kappa \eta}\right)^{n^{\prime}}$ that produces $n^{\prime} \triangleq\left\lfloor\frac{n}{\eta}\right\rfloor$ codevectors of size $\kappa \eta$; and

- two decoding mappings $g_{1}^{\left(n^{\prime}\right)}:\left(\mathbb{R}^{\nu_{1} \eta}\right)^{n^{\prime}} \rightarrow \mathcal{M}_{1}$ and $g_{2}^{\left(n^{\prime}\right)}:\left(\mathbb{R}^{\nu_{2} \eta}\right)^{n^{\prime}} \rightarrow \mathcal{M}_{2}$ that each decode a block of $n^{\prime}$ output vectors of lengths $\nu_{1} \eta$ and $\nu_{2} \eta$, respectively.

As already mentioned, the total blocklength $n$ is divided into $n^{\prime}$ subblocks of length $\eta$. The matrices $\left\{\mathrm{A}_{i, \tau, \ell}\right\}$ describe the inner code that is used within each of the $n^{\prime}$ subblocks. The parameters $f^{\left(n^{\prime}\right)}, g_{1}^{\left(n^{\prime}\right)}, g_{2}^{\left(n^{\prime}\right)}$ describe the outer code that is used to code over the $n^{\prime}$ subblocks without using the feedback.

We now describe how the inner code transforms the first block of $\eta$ channel uses into a single channel use of the new MIMO BC. All other blocks are transformed similarly. Let $\mathbf{X} \triangleq\left(\mathbf{X}_{1}^{\top}, \ldots, \mathbf{X}_{\eta}^{\top}\right)^{\top}, \mathbf{Z}_{i} \triangleq\left(\mathbf{Z}_{i, 1}^{\top}, \ldots, \mathbf{Z}_{i, \eta}^{\top}\right)^{\top}, \mathbf{Y}_{i} \triangleq$ $\left(\mathbf{Y}_{i, 1}^{\top}, \ldots, \mathbf{Y}_{i, \eta}^{\top}\right)^{\top}$, for $i \in\{1,2\}$, and let $\mathbf{W}$ denote the $\eta \kappa$ dimensional codevector produced by outer encoder $f^{\left(n^{\prime}\right)}$ for this first block. Define strictly-lower block-triangular matrices

$\mathrm{A}_{i}^{\mathrm{B}}=\left[\begin{array}{ccccc}0 & & \ldots & & 0 \\ \mathrm{~A}_{i, 1,2} & 0 & & & \\ \mathrm{~A}_{i, 1,3} & \mathrm{~A}_{i, 2,3} & 0 & & \\ \vdots & & & \ddots & \\ \mathrm{A}_{i, 1, \eta} & \mathrm{A}_{i, 2, \eta} & \ldots & \mathrm{A}_{i,(\eta-1), \eta} & 0\end{array}\right], i \in\{1,2\}$,

where here 0 denotes the $\kappa$-by- $\nu_{i}$ matrix with all zero entries, and the block channel-matrices $\mathrm{H}_{i}^{\mathrm{B}} \triangleq \mathrm{I}_{\eta} \otimes \mathrm{H}_{i}$, for $i \in\{1,2\}$, where $\otimes$ denotes the Kronecker product.

The channel inputs in the first block are chosen as

$$
\mathbf{X}=\left(\mathrm{I}-\mathrm{A}_{1}^{\mathrm{B}} \mathrm{H}_{1}^{\mathrm{B}}-\mathrm{A}_{2}^{\mathrm{B}} \mathrm{H}_{2}^{\mathrm{B}}\right) \mathbf{W}+\mathrm{A}_{1}^{\mathrm{B}} \mathbf{Y}_{1}+\mathrm{A}_{2}^{\mathrm{B}} \mathbf{Y}_{2} .
$$

Define further the matrices

$$
\mathrm{B}_{i}^{\mathrm{B}} \triangleq\left(\mathrm{I}-\mathrm{A}_{1}^{\mathrm{B}} \mathrm{H}_{1}^{\mathrm{B}}-\mathrm{A}_{2}^{\mathrm{B}} \mathrm{H}_{2}^{\mathrm{B}}\right)^{-1} \mathrm{~A}_{i}^{\mathrm{B}}, \quad i \in\{1,2\},
$$

after some algebraic manipulations, we can rewrite (12) as:

$$
\mathbf{X}=\mathbf{W}+\mathrm{B}_{1}^{\mathrm{B}} \mathbf{Z}_{1}+\mathrm{B}_{2}^{\mathrm{B}} \mathbf{Z}_{2}
$$

and the corresponding outputs as:

$$
\begin{aligned}
& \mathbf{Y}_{1}=\mathrm{H}_{1}^{\mathrm{B}} \mathbf{W}+\left(\mathrm{I}+\mathrm{H}_{1}^{\mathrm{B}} \mathrm{B}_{1}^{\mathrm{B}}\right) \mathbf{Z}_{1}+\mathrm{H}_{1}^{\mathrm{B}} \mathrm{B}_{2}^{\mathrm{B}} \mathbf{Z}_{2}, \\
& \mathbf{Y}_{2}=\mathrm{H}_{1}^{\mathrm{B}} \mathbf{W}+\left(\mathrm{I}+\mathrm{H}_{2}^{\mathrm{B}} \mathrm{B}_{2}^{\mathrm{B}}\right) \mathbf{Z}_{2}+\mathrm{H}_{2}^{\mathrm{B}} \mathrm{B}_{1}^{\mathrm{B}} \mathbf{Z}_{1} .
\end{aligned}
$$

Mapping $\left(\mathrm{A}_{1}^{\mathrm{B}}, \mathrm{A}_{2}^{\mathrm{B}}\right) \mapsto\left(\mathrm{B}_{1}^{\mathrm{B}}, \mathrm{B}_{2}^{\mathrm{B}}\right)$ in (13) is bijective and $\mathrm{B}_{i}^{\mathrm{B}}$, for $i \in\{1,2\}$, is strictly-lower block-triangular with blocks of sizes $\kappa \times \nu_{i}$.

By (14), the total input power in the first subblock is

$$
\mathrm{E}\left[\|\mathbf{X}\|^{2}\right]=\mathrm{E}\left[\|\mathbf{W}\|^{2}\right]+\operatorname{tr}\left(\mathrm{B}_{1}^{\mathrm{B}}\left(\mathrm{B}_{1}^{\mathrm{B}}\right)^{\mathrm{T}}\right)+\operatorname{tr}\left(\mathrm{B}_{2}^{\mathrm{B}}\left(\mathrm{B}_{2}^{\mathrm{B}}\right)^{\top}\right) .
$$

Since all the $n^{\prime}$ subblocks are constructed using the same $\mathrm{B}_{1}^{\mathrm{B}}$ and $\mathrm{B}_{2}^{\mathrm{B}}$, we conclude that the channel inputs $\left\{\mathbf{X}_{t}\right\}_{t=1}^{n}$ to our original MIMO BC satisfy the average block-power constraint (3) if $\operatorname{tr}\left(\mathrm{B}_{1}^{\mathrm{B}}\left(\mathrm{B}_{1}^{\mathrm{B}}\right)^{\mathrm{T}}\right)+\operatorname{tr}\left(\mathrm{B}_{2}^{\mathrm{B}}\left(\mathrm{B}_{2}^{\mathrm{B}}\right)^{\mathrm{T}}\right) \leq \eta P$ and if the codewords produced by the outer encoder $f^{\left(n^{\prime}\right)}$ are average block-power constrained to power

$$
\eta P-\operatorname{tr}\left(\mathrm{B}_{1}^{\mathrm{B}}\left(\mathrm{B}_{1}^{\mathrm{B}}\right)^{\top}\right)-\operatorname{tr}\left(\mathrm{B}_{2}^{\mathrm{B}}\left(\mathrm{B}_{2}^{\mathrm{B}}\right)^{\top}\right) .
$$

Definition 1. Let $\mathcal{R}_{\mathrm{BC}}\left(\eta, \mathrm{B}_{1}^{\mathrm{B}}, \mathrm{B}_{2}^{\mathrm{B}}, \mathrm{H}_{1}^{\mathrm{B}}, \mathrm{H}_{2}^{\mathrm{B}} ; P\right)$ denote the capacity region of the MIMO BC in (15) when the vector-input $\mathrm{W}$ is average block-power constrained to power (16).

Recall that our inner code transforms $\eta$ uses of the original MIMO BC into a single use of the new MIMO BC (15). Thus, if we design the outer code $\left\{f^{\left(n^{\prime}\right)}, g_{1}^{\left(n^{\prime}\right)}, g_{2}^{\left(n^{\prime}\right)}\right\}$ to achieve the nofeedback capacity of MIMO BC (15) under average input-power constraint (16) as described in [12], then over the original MIMO $\mathrm{BC}$ we achieve the rate region $\frac{1}{\eta} \mathcal{R}_{\mathrm{BC}}\left(\eta, \mathrm{B}_{1}^{\mathrm{B}}, \mathrm{B}_{2}^{\mathrm{B}}, \mathrm{H}_{1}^{\mathrm{B}}, \mathrm{H}_{2}^{\mathrm{B}} ; P\right)$. This proves the achievability part of the following proposition. The converse can be shown using Fano's inequality, and is omitted here due to lack of space.

\section{Proposition 1.}

$\mathcal{C}_{\mathrm{BC}}^{\operatorname{linfb}}\left(\mathrm{H}_{1}, \mathrm{H}_{2} ; P\right)=\mathrm{cl}\left(\bigcup_{\eta, \mathrm{B}_{1}^{\mathrm{B}}, \mathrm{B}_{2}^{\mathrm{B}}} \frac{1}{\eta} \mathcal{R}_{\mathrm{BC}}\left(\eta, \mathrm{B}_{1}^{\mathrm{B}}, \mathrm{B}_{2}^{\mathrm{B}}, \mathrm{H}_{1}^{\mathrm{B}}, \mathrm{H}_{2}^{\mathrm{B}} ; P\right)\right)$

where the union is over all positive integers $\eta$ and all strictlylower block-triangular $(\eta \kappa)$-by- $\left(\eta \nu_{1}\right)$ and $(\eta \kappa)$-by- $\left(\eta \nu_{2}\right)$ matrices $\mathrm{B}_{1}^{\mathrm{B}}$ and $\mathrm{B}_{2}^{\mathrm{B}}$ with blocks of sizes $\kappa \times \nu_{1}$ and $\kappa \times \nu_{2}$ that satisfy $\operatorname{tr}\left(\mathrm{B}_{1}^{\mathrm{B}}\left(\mathrm{B}_{1}^{\mathrm{B}}\right)^{\mathrm{T}}\right)+\operatorname{tr}\left(\mathrm{B}_{2}^{\mathrm{B}}\left(\mathrm{B}_{2}^{\mathrm{B}}\right)^{\mathrm{T}}\right) \leq \eta P$.

\section{B. A class of linear-feedback schemes for the MAC}

Fix the blocklength $n$. The schemes are parametrized by

- a positive integer $\eta$;

- $\nu_{i}$-by- $\kappa$ matrices $\left\{C_{i, \tau, \ell}\right\}$, for $\ell=2, \ldots, \eta, \tau=$ $1, \ldots, \ell-1$, and $i \in\{1,2\}$;

- two encoding mappings $f_{1}^{\left(n^{\prime}\right)}: \mathcal{M}_{1} \rightarrow\left(\mathcal{R}^{\nu_{1} \eta}\right)^{n^{\prime}}$ and $f_{2}^{\left(n^{\prime}\right)}: \mathcal{M}_{2} \rightarrow\left(\mathcal{R}^{\nu_{2} \eta}\right)^{n^{\prime}}$ that produce $n^{\prime}$ codevectors of sizes $\nu_{1} \eta$ and $\nu_{2} \eta$, respectively; and

- a decoding mapping $g^{\left(n^{\prime}\right)}:\left(\mathbb{R}^{\nu \eta}\right)^{n^{\prime}} \rightarrow \mathcal{M}_{1} \times \mathcal{M}_{2}$ that decodes a block of $n^{\prime}$ output vectors of length $\kappa \eta$.

The total blocklength is divided into $n^{\prime}$ subblocks of length $\eta$. The matrices $\left\{\mathrm{C}_{1, \tau, \ell}\right\}$ and $\left\{\mathrm{C}_{2, \tau, \ell}\right\}$ describe the inner code used within each of the $n^{\prime}$ subblocks of length $\eta$. The parameters $f_{1}^{\left(n^{\prime}\right)}, f_{2}^{\left(n^{\prime}\right)}, g^{\left(n^{\prime}\right)}$ describe the outer code used to code over the $n^{\prime}$ subblocks without using the feedback.

We describe the first block of $\eta$ channel inputs. The inputs to the other blocks are similar. For $i \in\{1,2\}$, let $\mathbf{X}_{i} \triangleq\left(\mathbf{X}_{i, 1}^{\top}, \ldots, \mathbf{X}_{i, \eta}^{\top}\right)^{\top}, \mathbf{Y} \triangleq\left(\mathbf{Y}_{1}^{\top}, \ldots, \mathbf{Y}_{\eta}^{\top}\right)^{\top}$, and $\mathbf{Z} \triangleq$ $\left(\mathbf{Z}_{1}^{\top}, \ldots, \mathbf{Z}_{\eta}^{\top}\right)^{\top}$ and let $\mathbf{U}_{i}$ denote the $\eta \nu_{i}$-length codevector (a column vector) produced by $f_{i}^{\left(n^{\prime}\right)}$ for this first block. Define the strictly-lower block-triangular matrices

$$
\mathrm{C}_{i}^{\mathrm{B}}=\left[\begin{array}{ccccc}
0 & & \ldots & & 0 \\
\mathrm{C}_{i, 1,2} & 0 & & & \\
\mathrm{C}_{i, 1,3} & \mathrm{C}_{i, 2,3} & 0 & & \\
\vdots & & & \ddots & \\
\mathrm{C}_{i, 1, \eta} & \mathrm{C}_{i, 2, \eta} & \ldots & \mathrm{C}_{i,(\eta-1), \eta} & 0
\end{array}\right], i \in\{1,2\},
$$


where here 0 denotes an $\nu_{i}$-by- $\kappa$ zero matrix. Transmitter $i$ 's inputs during the first block are chosen as

$$
\mathbf{X}_{i}=\mathrm{Q}_{i}^{-1} \mathbf{U}_{i}+\mathrm{C}_{i}^{\mathrm{B}} \mathbf{Y}
$$

Defining

$$
\mathrm{D}_{i}^{\mathrm{B}} \triangleq \mathrm{C}_{i}^{\mathrm{B}}\left(\mathrm{I}-\left(\mathrm{H}_{1}^{\mathrm{B}}\right)^{\top} \mathrm{C}_{1}^{\mathrm{B}}-\left(\mathrm{H}_{2}^{\mathrm{B}}\right)^{\top} \mathrm{C}_{2}^{\mathrm{B}}\right)^{-1}, i \in\{1,2\},
$$

and $Q_{1}$ and $Q_{2}$ as the unique positive square roots of the (positive-definite) matrices

$$
\begin{aligned}
& \mathrm{M}_{1} \triangleq\left(\mathrm{I}+\mathrm{D}_{1}^{\mathrm{B}}\left(\mathrm{H}_{1}^{\mathrm{B}}\right)^{\top}\right)^{\top}\left(\mathrm{I}+\mathrm{D}_{1}^{\mathrm{B}}\left(\mathrm{H}_{1}^{\mathrm{B}}\right)^{\top}\right)+\left(\mathrm{D}_{2}^{\mathrm{B}}\left(\mathrm{H}_{1}^{\mathrm{B}}\right)^{\top}\right)^{\top} D_{2}^{\mathrm{B}}\left(\mathrm{H}_{1}^{\mathrm{B}}\right)^{\top} \\
& \mathrm{M}_{2} \triangleq\left(\mathrm{I}+\mathrm{D}_{2}^{\mathrm{B}}\left(\mathrm{H}_{2}^{\mathrm{B}}\right)^{\top}\right)^{\top}\left(\mathrm{I}+\mathrm{D}_{2}^{\mathrm{B}}\left(\mathrm{H}_{2}^{\mathrm{B}}\right)^{\top}\right)+\left(\mathrm{D}_{1}^{\mathrm{B}}\left(\mathrm{H}_{2}^{\mathrm{B}}\right)^{\top}\right)^{\top} D_{1}^{\mathrm{B}}\left(\mathrm{H}_{2}^{\mathrm{B}}\right)^{\top},
\end{aligned}
$$

we can rewrite the inputs (18) and the corresponding outputs:

$$
\begin{aligned}
\mathbf{X}_{i}= & \mathrm{Q}_{i}^{-1} \mathbf{U}_{i}+\mathrm{D}_{i}^{\mathrm{B}}\left(\left(\mathrm{H}_{1}^{\mathrm{B}}\right)^{\top} \mathrm{Q}_{1}^{-1} \mathbf{U}_{1}+\left(\mathrm{H}_{2}^{\mathrm{B}}\right)^{\top} \mathrm{Q}_{2}^{-1} \mathbf{U}_{2}+\mathbf{Z}\right) \\
\mathbf{Y}=\left(\mathbf{I}+\left(\mathrm{H}_{1}^{\mathrm{B}}\right)^{\top} \mathrm{D}_{1}^{\mathrm{B}}+\left(\mathrm{H}_{2}^{\mathrm{B}}\right)^{\top} \mathrm{D}_{2}^{\mathrm{B}}\right) & \\
& \cdot\left(\left(\mathrm{H}_{1}^{\mathrm{B}}\right)^{\top} \mathrm{Q}_{1}^{-1} \mathbf{U}_{1}+\left(\mathrm{H}_{2}^{\mathrm{B}}\right)^{\top} \mathbf{Q}_{2}^{-1} \mathbf{U}_{2}+\mathbf{Z}\right) .
\end{aligned}
$$

Mapping $\left(\mathrm{C}_{1}^{\mathrm{B}}, \mathrm{C}_{2}^{\mathrm{B}}\right) \mapsto\left(\mathrm{D}_{1}^{\mathrm{B}}, \mathrm{D}_{2}^{\mathrm{B}}\right)$ in (19) is bijective and $\mathrm{D}_{i}^{\mathrm{B}}$, for $i \in\{1,2\}$, is strictly-lower block-triangular with blocks of sizes $\nu_{i} \times \kappa$.

Lemma 1. The channel inputs $\left\{\mathbf{X}_{1, t}\right\}_{t=1}^{n}$ and $\left\{\mathbf{X}_{2, t}\right\}_{t=1}^{n}$ to the original MIMO Gaussian MAC satisfy the total average input-power constraint (8), if $\operatorname{tr}\left(\mathrm{D}_{1}^{\mathrm{B}}\left(\mathrm{D}_{1}^{\mathrm{B}}\right)^{\mathrm{T}}\right)+\operatorname{tr}\left(\mathrm{D}_{2}^{\mathrm{B}}\left(\mathrm{D}_{2}^{\mathrm{B}}\right)^{\mathrm{T}}\right) \leq$ $\eta P$ and if the codevectors produced by $f_{1}^{\left(n^{\prime}\right)}$ and $f_{2}^{\left(n^{\prime}\right)}$ are total average block-power constrained to power

$$
\eta P-\operatorname{tr}\left(\mathrm{D}_{1}^{\mathrm{B}}\left(\mathrm{D}_{1}^{\mathrm{B}}\right)^{\mathrm{T}}\right)-\operatorname{tr}\left(\mathrm{D}_{2}^{\mathrm{B}}\left(\mathrm{D}_{2}^{\mathrm{B}}\right)^{\top}\right) .
$$

Proof: The proof is omitted due to lack of space.

Definition 2. Let $\mathcal{R}_{\mathrm{MAC}}\left(\eta, \mathrm{D}_{1}^{\mathrm{B}}, \mathrm{D}_{2}^{\mathrm{B}},\left(\mathrm{H}_{1}^{\mathrm{B}}\right)^{\top},\left(\mathrm{H}_{2}^{\mathrm{B}}\right)^{\top} ; P\right)$ denote the capacity region of the MIMO MAC in (22) under total average block-power constraint (23) on the inputs $\mathbf{U}_{1}$ and $\mathbf{U}_{2}$.

If we design the outer code $\left\{f_{1}^{\left(n^{\prime}\right)}, f_{2}^{\left(n^{\prime}\right)}, g^{\left(n^{\prime}\right)}\right\}$ so that it achieves the nofeedback capacity of the new MIMO MAC in (22) under total average input-power constraint (23), then over the original MIMO MAC we can achieve the rate region $\frac{1}{\eta} \mathcal{R}_{\mathrm{MAC}}\left(\eta, \mathrm{D}_{1}^{\mathrm{B}}, \mathrm{D}_{2}^{\mathrm{B}},\left(\mathrm{H}_{1}^{\mathrm{B}}\right)^{\mathrm{T}},\left(\mathrm{H}_{2}^{\mathrm{B}}\right)^{\top} ; P\right)$. This proves the achievability part of the following proposition. The converse is based on Fano's inequality and omitted here due to lack of space.

\section{Proposition 2.}

$$
\begin{aligned}
& \mathcal{C}_{\mathrm{MAC}}^{\text {linfb }}\left(\mathrm{H}_{1}^{\top}, \mathrm{H}_{2}^{\top} ; P\right) \\
& \quad=\operatorname{cl}\left(\bigcup_{\eta, \mathrm{D}_{1}^{\mathrm{B}}, \mathrm{D}_{2}^{\mathrm{B}}} \frac{1}{\eta} \mathcal{R}_{\mathrm{MAC}}\left(\eta, \mathrm{D}_{1}^{\mathrm{B}}, \mathrm{D}_{2}^{\mathrm{B}},\left(\mathrm{H}_{1}^{\mathrm{B}}\right)^{\top},\left(\mathrm{H}_{2}^{\mathrm{B}}\right)^{\top} ; P\right)\right)
\end{aligned}
$$

where the union is over all positive integers $\eta$ and all strictlylower block-triangular $\left(\eta \nu_{1}\right)$-by- $(\eta \kappa)$ and $\left(\eta \nu_{2}\right)$-by- $(\eta \kappa) m a$ trices $\mathrm{D}_{1}^{\mathrm{B}}$ and $\mathrm{D}_{2}^{\mathrm{B}}$ with blocks of sizes $\nu_{1} \times \kappa$ and $\nu_{2} \times \kappa$ that satisfy $\operatorname{tr}\left(\mathrm{D}_{1}^{\mathrm{B}}\left(\mathrm{D}_{1}^{\mathrm{B}}\right)^{\mathrm{T}}\right)+\operatorname{tr}\left(\mathrm{D}_{2}^{\mathrm{B}}\left(\mathrm{D}_{2}^{\mathrm{B}}\right)^{\mathrm{T}}\right) \leq \eta P$.

\section{Dual linear-feedback schemes for MAC and BC}

Definition 3. For a given $k$-by-l matrix $\mathrm{M}$, we define its reverse image as the $\ell$-by-k matrix $\overline{\mathrm{M}} \triangleq \mathrm{E}_{\ell} \mathrm{M}^{\top} \mathrm{E}_{k}$, where $\mathrm{E}_{k}$ denotes the $k$-by-k exchange matrix which is 0 everywhere except for the counter-diagonal where it is 1 . (In the following we often drop the subscript and simply write $\mathrm{E}$.)

Proposition 3. Let $\overline{\mathrm{H}}_{i}^{\mathrm{B}}=\mathrm{I}_{\eta} \otimes \overline{\mathrm{H}}_{i}$. If

$$
\mathrm{B}_{i}^{\mathrm{B}}=\overline{\mathrm{D}}_{i}^{\mathrm{B}}, \quad i \in\{1,2\},
$$

then the following two regions coincide:

$\mathcal{R}_{\mathrm{BC}}\left(\eta, \mathrm{B}_{1}^{\mathrm{B}}, \mathrm{B}_{2}^{\mathrm{B}}, \mathrm{H}_{1}^{\mathrm{B}}, \mathrm{H}_{2}^{\mathrm{B}} ; P\right)=\mathcal{R}_{\mathrm{MAC}}\left(\eta, \mathrm{D}_{1}^{\mathrm{B}}, \mathrm{D}_{2}^{\mathrm{B}}, \overline{\mathrm{H}}_{1}^{\mathrm{B}}, \overline{\mathrm{H}}_{2}^{\mathrm{B}} ; P\right)$.

Proof: See Section VI.

Condition (24) is equivalent to

$$
\mathrm{A}_{i}^{\mathrm{B}}=\overline{\mathrm{C}}_{i}^{\mathrm{B}} .
$$

Remark 1. On a MIMO MAC with channel matrices $\mathrm{H}_{1}$ and $\mathrm{H}_{2}$ the transmitters and receiver can mimic a MIMO MAC with channel matrices $\overline{\mathrm{H}}_{1}$ and $\overline{\mathrm{H}}_{2}$. It suffices that each transmitter multiplies (from the left) its input vectors by $\mathrm{E}$ before feeding them to the channel and that the receiver and the transmitters first multiply the output and feedback vectors by $\mathrm{E}$ (from the left) before further processing.

Combining Proposition 3, (26), and Remark 1 we obtain:

Corollary 1. Consider a MIMO BC with channel matrices $\left(\mathrm{H}_{1}, \mathrm{H}_{2}\right)$ and its dual MAC with channel matrices $\left(\mathrm{H}_{1}^{\top}, \mathrm{H}_{2}^{\top}\right)$. Fix the MAC-scheme parameters $\eta,\left\{\mathrm{C}_{1, \tau, \ell}\right\},\left\{\mathrm{C}_{2, \tau, \ell}\right\}, f_{1}^{\left(n^{\prime}\right)}$, $f_{2}^{\left(n^{\prime}\right)}$, and $g^{\left(n^{\prime}\right)}$. Choose now the $B C$-scheme parameters

$$
\mathrm{A}_{i, \tau, \ell}=\overline{\mathrm{C}}_{i, \eta-\tau, \eta-\ell+2},
$$

and an optimal outer code $f^{\left(n^{\prime}\right)}, g_{1}^{\left(n^{\prime}\right)}$, and $g_{2}^{\left(n^{\prime}\right)}$ as described in [12]. Then, our MAC and BC-schemes achieve the same rate regions.

In the SISO case, all conditions (27) are summarized by

$$
\mathrm{A}_{i}^{\mathrm{B}}=\overline{\mathrm{C}}_{i}^{\mathrm{B}} .
$$

\section{Main Results}

Theorem 1. $\mathcal{C}_{\mathrm{BC}}^{\operatorname{linfb}}\left(\mathrm{H}_{1}, \mathrm{H}_{2} ; P\right)=\mathcal{C}_{\mathrm{MAC}}^{\operatorname{linfb}}\left(\mathrm{H}_{1}^{\top}, \mathrm{H}_{2}^{\top} ; P\right)$.

Proof: Follows by Propositions 1, 2, and 3, and since Remark 1 implies $\mathcal{C}_{\mathrm{MAC}}^{\text {lintb }}\left(\mathrm{H}_{1}^{\top}, \mathrm{H}_{2}^{\top} ; P\right)=\mathcal{C}_{\mathrm{MAC}}^{\text {linfb }}\left(\overline{\mathrm{H}}_{1}, \overline{\mathrm{H}}_{2} ; P\right)$.

Corollary 2. $C_{\mathrm{BC}, \Sigma}^{\text {linfb }}\left(\mathrm{H}_{1}, \mathrm{H}_{2} ; P\right)=C_{\mathrm{MAC}, \Sigma}^{\text {linfb }}\left(\mathrm{H}_{1}^{\top}, \mathrm{H}_{2}^{\top} ; P\right)$.

Let $\mathcal{C}_{\mathrm{MAC}, \text { SISO }}^{\text {linf }}, \mathcal{C}_{\mathrm{MAC}, \text { SIMO }}^{\text {linf }}, \mathcal{C}_{\mathrm{MAC}, \text { MISO }}^{\text {linfb }}$ denote the linearfeedback capacity regions for the SISO, SIMO, and MISO Gaussian MAC with feedback. From the results by Ozarow [2] \& Jafar and Goldsmith [3] one readily obtains ${ }^{1}$ computable single-letter expressions for these linear-feedback capacity regions, which coincide with the true capacity regions for

\footnotetext{
${ }^{1}$ Ozarow and Jafar \& Goldsmith determined the capacity regions under individual power constraints for each transmitter. The capacity region under a total power constraint is obtained by taking the union of these capacity regions over all possible power splits.
} 
the respective setups. By Theorem 1, we thus obtain singleletter expressions for the linear-feedback capacity regions of the SISO, SIMO, and MISO Gaussian BC:

Corollary 3. Consider the SISO case where the channel matrices are scalars $h_{1}$ and $h_{2}$ :

$$
\mathcal{C}_{\mathrm{BC}, \mathrm{SISO}}^{\text {linfb }}\left(h_{1}, h_{2} ; P\right)=\mathcal{C}_{\mathrm{MAC}, \mathrm{SISO}}^{\text {linfb }}\left(h_{1}, h_{2} ; P\right),
$$

where a computable single-letter characterization of $\mathcal{C}_{\text {MAC,SISO }}^{\text {linf }}\left(h_{1}, h_{2} ; P\right)$ is obtained from Ozarow's result [2].

Corollary 4. Consider the SIMO and MISO cases where the channel matrices are given by column vectors $\mathbf{h}_{1}$ and $\mathbf{h}_{2}$ :

$$
\begin{aligned}
\mathcal{C}_{\mathrm{BC}, \mathrm{MISO}}^{\text {linfb }}\left(\mathbf{h}_{1}^{\top}, \mathbf{h}_{2}^{\top} ; P\right) & =\mathcal{C}_{\mathrm{MAC}, \text { SIMO }}^{\text {linfb }}\left(\mathbf{h}_{1}, \mathbf{h}_{2} ; P\right) \\
\mathcal{C}_{\mathrm{BC}, \text { SIMO }}^{\text {linfb }}\left(\mathbf{h}_{1}, \mathbf{h}_{2} ; P\right) & =\mathcal{C}_{\text {MAC,MISO }}^{\text {linfb }}\left(\mathbf{h}_{1}^{\top}, \mathbf{h}_{2}^{\top} ; P\right) \\
& =\mathcal{C}_{\text {MAC,SISO }}^{\text {linfb }}\left(\left\|\mathbf{h}_{1}\right\|,\left\|\mathbf{h}_{2}\right\| ; P\right),
\end{aligned}
$$

where the last equality follows from a result in [3]. A computable single-letter characterization of $\mathcal{C}_{\mathrm{MAC}, \mathrm{SIMO}}^{\mathrm{linb}}$ and $\mathcal{C}_{\mathrm{MAC}, \mathrm{MISO}}^{\mathrm{linf}}$ can be obtained from the results in [3].

In view of Corollary 1 and the MAC capacity-achieving schemes in [2] and [3], for the SISO, the SIMO, and the MISO BC we can easily deduce the parameters of our linearfeedback schemes in Section IV-A that achieve the linearfeedback capacity.

Remark 2. Analagous results also hold with partial feedback where only a subset of the feedback links are present.

\section{Proof of Proposition 3}

Fix $\eta$ and strictly-lower block-triangular matrices $\mathrm{B}_{1}^{\mathrm{B}}, \mathrm{B}_{2}^{\mathrm{B}}$. Also, let $D_{1}^{\mathrm{B}}, \mathrm{D}_{2}^{\mathrm{B}}$ be given by (24). (Since $\mathrm{B}_{1}^{\mathrm{B}}$ and $\mathrm{B}_{2}^{\mathrm{B}}$ are strictly-lower block-triangular, so are $\mathrm{D}_{1}^{\mathrm{B}}$ and $\mathrm{D}_{2}^{\mathrm{B}}$.)

Consider the MIMO MAC (22) with $\left(\mathrm{H}_{i}^{\mathrm{B}}\right)^{\top}$ replaced by $\overline{\mathrm{H}}_{i}^{\mathrm{B}}$ :

$$
\mathbf{Y}^{\prime}=\left(\mathrm{I}+\overline{\mathrm{H}}_{1}^{\mathrm{B}} \mathrm{D}_{1}^{\mathrm{B}}+\overline{\mathrm{H}}_{2}^{\mathrm{B}} \mathrm{D}_{2}^{\mathrm{B}}\right)\left(\overline{\mathrm{H}}_{1}^{\mathrm{B}} \mathrm{Q}_{1}^{-1} \mathbf{U}_{1}+\overline{\mathrm{H}}_{2}^{\mathrm{B}} \mathrm{Q}_{2}^{-1} \mathbf{U}_{2}+\mathbf{Z}\right)
$$

where now $Q_{1}$ and $Q_{2}$ are the unique positive square-roots of

$$
\begin{aligned}
& \mathrm{M}_{1}=\left(\mathrm{I}+\mathrm{D}_{1}^{\mathrm{B}} \overline{\mathrm{H}}_{1}^{\mathrm{B}}\right)^{\top}\left(\mathrm{I}+\mathrm{D}_{1}^{\mathrm{B}} \overline{\mathrm{H}}_{1}^{\mathrm{B}}\right)+\left(\mathrm{D}_{2}^{\mathrm{B}} \overline{\mathrm{H}}_{1}^{\mathrm{B}}\right)^{\top}\left(\mathrm{D}_{2}^{\mathrm{B}} \overline{\mathrm{H}}_{1}^{\mathrm{B}}\right), \\
& \mathrm{M}_{2}=\left(\mathrm{I}+\mathrm{D}_{2}^{\mathrm{B}} \overline{\mathrm{H}}_{2}^{\mathrm{B}}\right)^{\top}\left(\mathrm{I}+\mathrm{D}_{2}^{\mathrm{B}} \overline{\mathrm{H}}_{2}^{\mathrm{B}}\right)+\left(\mathrm{D}_{1}^{\mathrm{B}} \overline{\mathrm{H}}_{2}^{\mathrm{B}}\right)^{\top}\left(\mathrm{D}_{1}^{\mathrm{B}} \overline{\mathrm{H}}_{2}^{\mathrm{B}}\right) .
\end{aligned}
$$

Since $\left(I+\bar{H}_{1}^{B} D_{1}^{B}+\bar{H}_{2}^{B} D_{2}^{B}\right)$ is invertible, under any given input power constraint, MAC (33) has the same capacity as MAC

$$
\mathbf{Y}_{\text {MAC }}^{\prime} \triangleq \overline{\mathrm{H}}_{1}^{\mathrm{B}} \mathrm{Q}_{1}^{-1} \mathbf{U}_{1}+\overline{\mathrm{H}}_{2}^{\mathrm{B}} \mathrm{Q}_{2}^{-1} \mathbf{U}_{2}+\mathbf{Z} \text {. }
$$

We now turn to $B C$ (15). Let $S_{1}$ and $S_{2}$ be the positive square roots of

$$
\begin{aligned}
& \mathrm{N}_{1} \triangleq\left(\mathrm{I}+\mathrm{H}_{1}^{\mathrm{B}} \mathrm{B}_{1}^{\mathrm{B}}\right)\left(\mathrm{I}+\mathrm{H}_{1}^{\mathrm{B}} \mathrm{B}_{1}^{\mathrm{B}}\right)^{\top}+\left(\mathrm{H}_{1}^{\mathrm{B}} \mathrm{B}_{2}^{\mathrm{B}}\right)\left(\mathrm{H}_{1}^{\mathrm{B}} \mathrm{B}_{2}\right)^{\top} \\
& \mathrm{N}_{2} \triangleq\left(\mathrm{H}_{2}^{\mathrm{B}} \mathrm{B}_{1}^{\mathrm{B}}\right)\left(\mathrm{H}_{2}^{\mathrm{B}} \mathrm{B}_{1}^{\mathrm{B}}\right)^{\top}+\left(\mathrm{I}+\mathrm{H}_{2}^{\mathrm{B}} \mathrm{B}_{2}^{\mathrm{B}}\right)\left(\mathrm{I}+\mathrm{H}_{2}^{\mathrm{B}} \mathrm{B}_{2}^{\mathrm{B}}\right)^{\top} .
\end{aligned}
$$

Define $\tilde{\mathbf{W}} \triangleq \mathrm{EW}$ and notice $\|\tilde{\mathbf{W}}\|^{2}=\|\mathbf{W}\|^{2}$. Since multiplying the output vectors of a MIMO BC by invertible matrices (here $\mathrm{ES}_{1}^{-1}$ and $\mathrm{ES}_{2}^{-1}$ ) does not change capacity, we conclude: The MIMO BC (15) and the MIMO BC

$$
\mathbf{Y}_{i, \mathrm{BC}}^{\prime} \triangleq \mathrm{ES}_{i}^{-1} \mathrm{H}_{i}^{\mathrm{B}} \mathrm{E} \tilde{\mathbf{W}}+\tilde{\mathbf{Z}}_{i}, \quad i \in\{1,2\},
$$

where $\tilde{\mathbf{Z}}_{1}$ and $\tilde{\mathbf{Z}}_{2}$ are independent centered Gaussian vectors of identity covariance matrices, have equal capacity when the inputs $\mathbf{W}$ and $\tilde{\mathbf{W}}$ are average block-power constrained to (16).

We conclude the proof by showing that the capacity regions of the MIMO BC in (37) under power constraint (16) and the MIMO MAC (35) under power constraint (23) coincide. In view of (24), comparing (34) and (36), we have

$$
\mathrm{EM}_{i} \mathrm{E}=\mathrm{N}_{i}, \quad i \in\{1,2\},
$$

and thus by the definitions and uniqueness of $S_{i}$ and $Q_{i}$,

$$
\mathrm{S}_{i}=\mathrm{EQ}_{i} \mathrm{E}=\mathrm{EQ}_{i}^{\top} \mathrm{E}, \quad i \in\{1,2\},
$$

where we used the symmetry of $Q_{i}$. This implies

$$
\mathrm{ES}_{i}^{-1} \mathrm{H}_{i}^{\mathrm{B}} \mathrm{E}=\mathrm{Q}_{i}^{-\top} \mathrm{EH}_{i}^{\mathrm{B}} \mathrm{E}=\left(\overline{\mathrm{H}}_{i}^{\mathrm{B}} \mathrm{Q}_{i}^{-1}\right)^{\top}, \quad i \in\{1,2\},
$$

and the BC (37) and the MAC (35) are duals and have therefore the same capacity regions under the same total power constraints on the inputs [10], [11]. Since by (24), the powers (16) and (23) are the same, this concludes the proof.

\section{ACKNOWLEDGEMENTS}

The work of S. Belhadj Amor and M. Wigger has been supported by the city of Paris under the "Emergences" program. The work of Y. Steinberg has been supported by the Israel Science Foundation (grant no. 684/11).

\section{REFERENCES}

[1] E. Ardestanizadeh, M. Wigger, Y. H. Kim, and T. Javidi, "Linearfeedback sum-capacity for Gaussian multiple access channels," IEEE Trans. on Inf. Th., vol. 58, no. 1, pp. 224-236, 2012.

[2] L. Ozarow, "The capacity of the white Gaussian multiple access channel with feedback," IEEE Trans. on Inf. Th., vol. 30, no. 4, pp. 623-629, 1984.

[3] S. Jafar and A. Goldsmith, "On the capacity of the vector MAC with feedback,” IEEE Trans. on Inf. Th., vol. 52, no. 7, pp. 3259-3264, 2006.

[4] G. Kramer, "Feedback strategies for white Gaussian interference networks," IEEE Trans. on Inf. Th., vol. 48, no. 6, pp. 1423-1438, 2002.

[5] L. Ozarow and S. Leung-Yan-Cheong, "An achievable region and outer bound for the Gaussian broadcast channel with feedback," IEEE Trans. on Inf. Th., vol. 30, no. 4, pp. 667-671, 1984.

[6] N. Elia, "When Bode meets Shannon: control-oriented feedback communication schemes," IEEE Trans. Automat. Contr., vol. 49, no. 9, pp. 1477-1488, 2004.

[7] S. Vishwanath, W. Wu, and A. Arapostathis, "Gaussian interference networks with feedback: duality, sum capacity and dynamic team problems," in Proc. 43rd Ann. Allerton Conf. , pp. 926-935, 2005.

[8] E. Ardestanizadeh, P. Minero, and M. Franceschetti, "LQG control approach to Gaussian broadcast channels with feedback," IEEE Trans. on Inf. Th., vol. 58, no. 8, pp. 5267-5278, 2012.

[9] M. Gastpar, A. Lapidoth, Y. Steinberg, and M. Wigger, "Coding schemes and asymptotic capacity for the Gaussian broadcast and interference channels with feedback," IEEE Trans. on Inf. Th., vol. 60, no. 1, pp. 5471,2014

[10] S. Vishwanath, N. Jindal, and A. Goldsmith, "Duality, achievable rates, and sum-rate capacity of Gaussian MIMO broadcast channels," IEEE Trans. on Inf. Theory, vol. 49, no. 10, pp. 2658-2668, 2003.

[11] P. Viswanath and D.N.C. Tse, "Sum capacity of the vector Gaussian broadcast channel and uplink-downlink duality," IEEE Trans. on Inf. Theory, vol. 49, no. 8, pp. 1912-1921, 2003.

[12] H. Weingarten, Y. Steinberg, and S. Shamai, "The capacity region of the Gaussian multiple-input multiple-output broadcast channel," IEEE Trans. on Inf. Theory, vol. 52, no. 9, pp. 3936-3964, 2006.

[13] S. Belhadj Amor, Y. Steinberg, and M. Wigger, "Duality with linearfeedback schemes for the scalar Gaussian MAC and BC," in Proceedings of IZS, pp. 25-28, 2014. 